OPEN ACCESS

Edited by:

Jin Y. Ro,

University of Maryland, Baltimore,

United States

Reviewed by:

Jan Mens,

Erasmus University

Rotterdam, Netherlands

Armen N. Akopian,

The University of Texas Health Science Center at San Antonio,

United States

${ }^{*}$ Correspondence:

Catherine Daneau

catherine.daneau@uqtr.ca

Specialty section

This article was submitted to

Musculoskeletal Pain

a section of the journal

Frontiers in Pain Research

Received: 10 September 2021

Accepted: 11 November 2021

Published: 02 December 2021

Citation:

Daneau C, Abboud J, Marchand A-A

Houle $M$, Pasquier $M$, Ruchat $S-M$

and Descarreaux M (2021)

Mechanisms Underlying Lumbopelvic

Pain During Pregnancy: A Proposed

Model. Front. Pain Res. 2:773988.

doi: 10.3389/fpain.2021.773988

\section{Mechanisms Underlying Lumbopelvic Pain During Pregnancy: A Proposed Model}

\author{
Catherine Daneau ${ }^{1 *}$, Jacques Abboud ${ }^{2}$, Andrée-Anne Marchand ${ }^{3}$, Mariève Houle ${ }^{1}$, \\ Mégane Pasquier ${ }^{1}$, Stephanie-May Ruchat ${ }^{2}$ and Martin Descarreaux ${ }^{2}$ \\ ${ }^{1}$ Department of Anatomy, Université du Québec à Trois-Rivières, Trois-Rivières, QC, Canada, ${ }^{2}$ Department of Human \\ Kinetics, Université du Québec à Trois-Rivières, Trois-Rivières, QC, Canada, ${ }^{3}$ Department of Chiropractic, Université du \\ Québec à Trois-Rivières, Trois-Rivières, QC, Canada
}

Up to $86 \%$ of pregnant women will have lumbopelvic pain during the 3rd trimester of pregnancy and women with lumbopelvic pain experience lower health-related quality of life during pregnancy than women without lumbopelvic pain. Several risk factors for pregnancy-related lumbopelvic pain have been identified and include history of low back pain, previous trauma to the back or pelvis and previous pregnancy-related pelvic girdle pain. During pregnancy, women go through several hormonal and biomechanical changes as well as neuromuscular adaptations which could explain the development of lumbopelvic pain, but this remains unclear. The aim of this article is to review the potential pregnancy-related changes and adaptations (hormonal, biomechanical and neuromuscular) that may play a role in the development of lumbopelvic pain during pregnancy. This narrative review presents different mechanisms that may explain the development of lumbopelvic pain in pregnant women. A hypotheses-driven model on how these various physiological changes potentially interact in the development of lumbopelvic pain in pregnant women is also presented. Pregnancy-related hormonal changes, characterized by an increase in relaxin, estrogen and progesterone levels, are potentially linked to ligament hyperlaxity and joint instability, thus contributing to lumbopelvic pain. In addition, biomechanical changes induced by the growing fetus, can modify posture, load sharing and mechanical stress in the lumbar and pelvic structures. Finally, neuromuscular adaptations during pregnancy include an increase in the activation of lumbopelvic muscles and a decrease in endurance of the pelvic floor muscles. Whether or not a causal link between these changes and lumbopelvic pain exists remains to be determined. This model provides a better understanding of the mechanisms behind the development of lumbopelvic pain during pregnancy to guide future research. It should allow clinicians and researchers to consider the multifactorial nature of lumbopelvic pain while taking into account the various changes and adaptations during pregnancy.

Keywords: hormonal changes, biomechanical changes, neuromuscular adaptations, motor control, pain modulation 


\section{INTRODUCTION}

Pregnancy-related low back pain (LBP) and/or pelvic girdle pain (PGP) are very common conditions, affecting up to $86 \%$ of pregnant women in the 3rd trimester of pregnancy (1). LBP is defined as "pain or discomfort located between the 12th rib and the gluteal fold," and PGP as "pain experienced between the posterior iliac crest and the gluteal fold" (2). When both types of pain are present, the pain is frequently referred to as lumbopelvic pain (LBPP). Despite variation in definitions, there seems to be a consensus that the term LBPP is used when no distinction is made between LBP and PGP (3). The peak of LBPP intensity generally appears between 24 and 36 weeks of pregnancy (4). Women suffering from LBPP during pregnancy will still experience LBPP beyond 3 months [33\% (5)] and 12 months $[25 \%(3,6)]$ after delivery.

Usually, women experience a decrease in health-related quality of life during pregnancy, but the decrease is greater for those with LBP (7). Indeed, pregnancy-related LBPP has important consequences on daily functioning and overall wellbeing (8-11). Importantly, LBPP has been found to contribute to high levels of sick leave in pregnant women (8).

\section{HYPOTHESIS}

Several potential risk factors for pregnancy-related LBP or PGP have been suggested; most of them being drawn from observational studies. Based on the exhaustive review of Verstraete et al. (12), history of LBP, previous trauma to the back or pelvis and previous pregnancy-related PGP seem to be strong risk factors for PGP during pregnancy. Two large population studies $n=74973$ (13) and $n=91721$ (14) also found that early menarche ( $\leq 13$ years old), low maternal age ( $<35$ years old), high body mass index $\left(\geq 25 \mathrm{~kg} / \mathrm{m}^{2}\right)$, parity, low educational level ( $\leq 16$ years of education), the presence of LBP before the first pregnancy, emotional distress, physically demanding work and the use of oral contraceptives were associated with increased odds for PGP during pregnancy. More specifically regarding oral contraception, Bjelland et al. (14) reported that the association between combined oral contraceptive pills and PGP during pregnancy was negative for primiparae (slightly protective effect of combined oral contraceptives) and positive for multiparae (marginally increased risk of PGP). Based on sub-analyses, the authors also concluded that long duration of exposure to a progestin intrauterine device or progestinonly oral contraceptives was associated with reduced odds of persistent pelvic girdle pain (Ptrend $=0.021$ and Ptrend $=0.005$, respectively). Conversely, long duration of exposure to progestin injections and/or a progestin implant was associated with modest increased odds of persistent pelvic girdle pain (Ptrend $=0.046$ ). Finally, the authors reported that early timing of progestin-only contraceptive dispense following delivery ( $<3$ months) was not significantly associated with persistent pelvic girdle pain (14).

Lack of exercise in mid-pregnancy has also been suggested to be associated with higher prevalence of LBP or PGP in late pregnancy (15).
Despite the high prevalence of pregnancy-related LBPP and its significant impact on women's quality of life, the physiological and biomechanical processes underlying the development of pregnancy-related LBPP remain unclear. This might explain why optimal management strategies that are based on underlying pathophysiological mechanisms are still lacking. In this hypothesis paper, we highlight several potential pregnancyrelated changes (hormonal and biomechanical) and adaptations (neuromuscular) and propose a hypotheses-driven model describing how these various physiological and biomechanical changes potentially interact in the development of LBPP in pregnant women. A better understanding of the mechanisms behind the development of LBPP during pregnancy should guide future research and lead to more effective management.

\section{LUMBOPELVIC PAIN UNDERLYING MECHANISMS}

\section{Hormonal Changes}

Pregnancy is characterized by significant hormonal changes, such as an increase in relaxin, estrogen and progesterone levels, and it has been hypothesized that these pregnancy-related hormones are potential contributors to LBPP development and intensity (16).

In pregnant women, relaxin levels increase by the end of the 1 st trimester and remain relatively high until delivery (16). The role of relaxin is to relax spinal and pelvic ligaments and joints in order to facilitate childbirth. Relaxin has been shown to increase the laxity of the pubic ligaments in guinea pigs (17). A study showed that female guinea pigs that received hormonal treatment (relaxin or relaxin + estrogen) had weaker anterior cruciate ligaments (ACL), as reflected by a lower load capacity before failure for both hormonal treatments (compared to no treatment) and an increase in tibial displacement (compared to baseline), potentially suggesting knee instability (18). A review on the effect of relaxin on human and animal musculoskeletal structures, such as ligaments, suggested that relaxin could predispose the joints to non-traumatic injury via its effect on peripheral ligament laxity (19). As such, it has been suggested that relaxin may trigger spinal and pelvic instability, potentially inducing pain $(19,20)$. However, based on the findings of a systematic review, the association between relaxin levels and pregnancy-related LBPP is inconsistent and the quality level of the evidence is poor (21).

Compared to relaxin, studies investigating the effect of estrogen and progesterone on ligaments property and joint instability are conflicting. During pregnancy, estrogen levels raise steeply during the 3rd trimester (22). In addition to promoting fetal growth and well-being, estrogen has an effect on several properties of the musculoskeletal tissue such as bone, cartilage and ligaments, modulates the nervous system and potentially contributes to LBPP development and intensity (16). A study conducted on human ACL specimens showed that the higher the estrogen levels, the lower the fibroblasts proliferation and synthesis of procollagen, possibly increasing ligament laxity (23). This correlation was attenuated with increased progesterone levels (23). It has also been shown that high estrogen levels in 
the 3rd trimester of human pregnancy were correlated with an increase in anterior translation of the tibia, suggesting an increase in ACL laxity (24). However, a study reported that estrogen levels were not different between women reporting PGP and those not reporting such pain (4).

Finally, progesterone levels increase significantly throughout pregnancy, with a peak at $\sim 15$ weeks (25). This hormone contributes to the relaxation of all smooth muscles during pregnancy (22), with a possible effect on LBPP development and intensity (23). To the best of our knowledge, only one study investigated the possible association between progesterone and LBPP and reported that progesterone levels were significantly higher in the 1st trimester of pregnancy ( 6 to 12 weeks) among women reporting PGP compared to those reporting no pain (4).

Levels of prolactin and oxytocin also fluctuate during pregnancy and postpartum period. Prolactin, which increases in the 1st trimester, is 10 time higher at the end of pregnancy (26) and plays a major role in stimulating maternal milk production (27). Oxytocin level increases during pregnancy and reaches its peak at the very end (26). Oxytocin is a stimulator of uterine contractions during childbirth (27).

Based on current evidence, relaxin, estrogen, and progesterone may play a role in the development and intensity of pregnancy-related LBPP but further studies are needed to confirm this hypothesis. The role of prolactin and oxytocin remains to be investigated. The strength of correlation between hormonal status and LBPP development and intensity should be investigated through cohort studies.

\section{Biomechanical Changes}

Profound biomechanical changes occur over the course of pregnancy (28). However, only a few studies investigated the association between these changes and LBPP. The most important pregnancy-related changes occur in the lower trunk and pelvic areas in response to the fetal load (29). Different kyphosis and lordosis configurations (30-37), as well as pelvic positions $(30,38,39)$, have been described in response to the change in magnitude and distribution of loads acting on the spine during pregnancy. The overall increase in lumbar lordosis is generally heterogeneous and inconstant across studies. The post-partum period is also marked with variable modifications to spinal curvatures $(33,40-42)$ that appear to be independent of the ones observed during pregnancy. Despite this heterogeneity and inconsistency, most pregnant women experience a small absolute increase in lumbar lordosis and in LBP intensity, most commonly during the 2nd and 3rd trimesters, when the size of the fetus increases significantly and the hormonal milieu changes markedly $(31,33,35,43)$. Although the increase in lumbar lordosis angle and in LBP intensity follow parallel trajectories throughout pregnancy, the causality in this relationship remains unclear.

Several studies have examined the association between prepregnancy joint hypermobility and LBPP during pregnancy, with mixed results (44-47). Some studies reported an association (4547), with an early study (47) showing a very strong relation between degree of symphyseal laxity and the risk for pelvic girdle pain during post-partum. However, a study with smaller sample size, found no association (44). Other authors suggested that pain could be explained by an asymmetric laxity of the sacro-iliac joints rather than by an increase in joint laxity per se (48). The joint laxity hypothesis is further emphasized by the fact that a possible cumulative effect exists as multiparity is a risk factor for PGP during pregnancy $(13,49)$.

Furthermore, sustained strain of pelvic structures and muscle weakness are believed to decrease mechanical force closure of the sacro-iliac joints, negatively influencing load transfer (50). Such proposed reduction in load transfer abilities are coherent with findings of altered gait pattern, including longer double limb support, shorter step length and less pelvic and hip movement, in women with pregnancy-related PGP (51-53). As previously mentioned, previous trauma to the back or pelvis area have been reported to be strong predictors of LBPP during pregnancy $(12,49)$ potentially leading to muscular imbalances and joint misalignments, which in turn could be aggravated by pregnancy-related anatomical changes (49). Finally, pregnancyrelated morphological changes related to the bone structures of the pelvis, including increased pubic symphysis width and inter-ischial tuberosity distance, have been proposed as potential contributors to PGP (54-56).

\section{Neuromuscular Adaptations}

Evidence suggesting motor control and neuromuscular adaptations during pregnancy and a possible link with LBPP is scarce, but some "patterns" of adaptations seem to be more consistent across studies (54). Several studies have identified an increased activation of lumbopelvic muscles during pregnancy. During a trunk flexion-extension task, pregnant women without LBPP show a significant increase in erector spinae muscle activation during the active flexion and the static full flexion phases of movement (57). Erector spinae and biceps femoris activity also seems to increase in upright posture in pregnant women compared to non-pregnant women (40). Compared to pregnant women without LBPP, those with LBPP have increased rectus femoris, abdominal obliques, psoas major, and adductor longus activities during active straight leg raising test (ASLR) (58) as well as increased intravaginal muscle activation during simulated pushing contractions (59). Stuge et al. (60) also reported a smaller levator hiatus (a proxy measure of pelvic floor muscle activation) at rest, during voluntary contractions and contraction associated to ASLR in women with PGP compared to those without PGP. Interestingly, an early study showed that pain intensity and erector spinae muscle activation were correlated in the 1st trimester of pregnancy, potentially suggesting a dose-response relationship (61).

Despite no clear evidence of neuromuscular manifestation of muscle fatigue, decrease endurance of the pelvic floor muscles, as well as an increased perineal tonus, have been reported in association with pregnancy-related PGP $(50,59,62)$. Moreover, women with PGP and/or combined PGP and LBP were shown to have lower trunk muscle endurance (52).

Interestingly, two recently published studies $(40,57)$ reported that changes in trunk and lower limb muscle activation during upright standing and flexion-extension movements of the trunk are similar before and after pregnancy, suggesting progressive 
short-term neuromuscular adaptations during pregnancy that appear to rapidly fade following delivery. Reversibility in neuromuscular adaptations that occur during pregnancy is also supported by an earlier study indicating that reduced trunk ranges of motion during pregnancy return to pre-pregnancy values during the postpartum period (57).

\section{Pain Modulation}

Central and peripheral sensitization have both been suggested to play a role in chronic pain conditions such as non-specific LBP and may increase the risk of a persistent pain. Physiological mechanisms leading to enhanced pain sensitivity have been thoroughly investigated and include sensitization of nociceptors and neuronal circuits (63), increased pain signaling through membrane excitability and synaptic efficacy, and inadequate descending pain inhibition mechanisms $(64,65)$. Whether or not these pain sensitization mechanisms play a significant role in the development and persistence of LBPP remains to be determined.

It has been consistently reported that women and men experience pain differently $(66,67)$. Women usually have a lower pain threshold and the cortical regions responsible for the affective component of pain show a higher brain activity (68). Prolonged pain can lead to central sensitization, which is also more present in women and mainly observed in women suffering from deep tissue pain (68). Differences in hormone levels between men and women represent the most important factor of these pain modulation disparities.

During pregnancy, changes in sex hormones, and the immune system improves to support the growing fetus (69). Sex hormones seem to play a role in pain modulation. Indeed, in non-pregnant women, it has been shown that an increase in estrogen levels correlates with a higher risk of LBP occurrence (70). During pregnancy, estrogen levels raise and have been hypothesized to contribute to LBPP development and intensity. Nevertheless, it has been suggested that hormone variations in pregnant women cannot be considered the sole contributor to anti-nociceptive or pro-nociceptive responses.

Interestingly, in female already living with chronic pain before becoming pregnant, an attenuation of pain symptoms has been observed during pregnancy. This adaptation refers to the pregnancy-induced analgesia phenomenon. This phenomenon has been reported for acute, as well as chronic, pain in pregnant animals and is dependent on various factors, such as variation in pregnancy-related hormone levels, the activation of cells of the immune system ( $\mathrm{T}$ cells), and the release of $\delta$-opioid (71, 72). However, the pregnancy-induced analgesia phenomenon is not always observed in humans. Studies have highlighted a progressive increase in pain threshold and tolerance in pregnant women compared to non-pregnant women $(73,74)$ while other studies failed to find such a pain adaptation (74-78). It has also been reported that widespread deep-tissue pressure hypersensitivity is higher and increased in pregnant women who experienced either low or high levels of LBPP compared to painfree non-pregnant women (79). The discrepancy between animal and human studies on pain modulation could be explained by different factors, such as differences in pain threshold, location of the painful stimulus, age, medication and psychological aspects [fear-avoidance and catastrophizing of pain $(80,81)$ ] which are more prevalent in humans (82).

At 4 to 8 weeks after delivery, an increase in hormone levels (i.e., estrogen and progesterone) has been observed in comparison to the last weeks of pregnancy (83). However, this hormone change does not seem to affect the pain modulation as similar heat pain threshold and tolerance in the upper limb were observed in postpartum women compared to pregnant (75) [and non-pregnant women (84)]. Yet, another study showed that postpartum women were more sensitive to pain at the rectus abdominis muscle than non-pregnant women (85). Conversely, lower capsaicin-evoked upper limb pain has been shown in postpartum women compared to non-pregnant women (84). Such postpartum analgesic phenomenon may be explained by oxytocin released into the systemic circulation and central nervous system, as evidenced by increased concentrations in cerebrospinal fluid during labor (86). Oxytocin may therefore play a role in pain modulation through reduced sensitization or enhanced inhibition mechanisms (84).

\section{DISCUSSION}

The past decades have seen a significant growth in mechanistic research aimed at investigating the physiological processes involved in the development and chronification of LBP. Although uncertainties persist, our understanding of biomechanical, motor control and neuromuscular adaptations to LBP has greatly improved and we now know that changes in motor behavior associated to LBP involve complex $(87,88)$ and most likely non-stereotypical "adaptive strategies" (89). Overall, LBP triggers adaptations at multiple levels of the motor system (87) which involves a redistribution of muscular activity within and between muscles as well as numerous changes in mechanical behaviors. According to "the new pain adaptation theory" proposed by Hodges and Tucker, and largely inspired by LBP research, the changes observed in the motor system occur in response to pain and are tailored to protect from further pain or re-injury, but also entail possible long-term adverse consequences. Whether pregnancy-related LBPP and non-specific LBP share similar underlying physiological mechanisms is still up for debate but the fact that pregnant women go through several physical and biomechanical adaptations in a relatively short period of time is unmistakable.

Similarly to LBP, LBPP is a complex and multifaceted condition for which underlying mechanisms remain elusive. The development and evolution of LBPP during and following pregnancy are probably dictated by complex interactions between risks factors, physiological processes and other contextual factors. The following theoretical model will hopefully provide some insights into these complex interactions and guide the design and interpretation of both mechanistic and clinical research. The model, shown in Figure 1, postulates that every pregnant woman can have a varying number of LBPP risk factors. During pregnancy, women will undergo several changes and adaptations that can be potentially mediated by other individual characteristics and biological mechanisms 


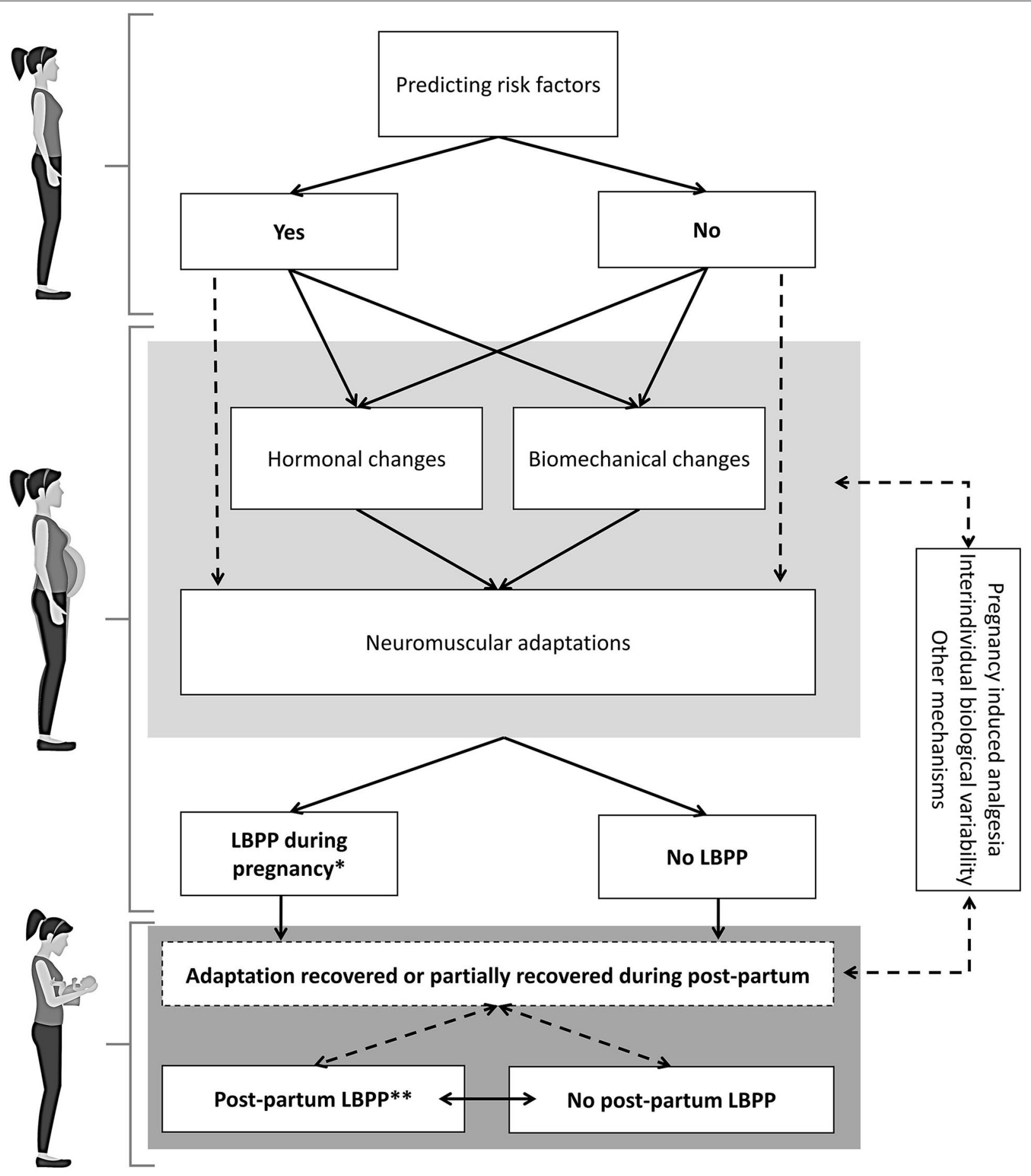

FIGURE 1 | Lumbopelvic pain (LBPP) underlying mechanisms. This model presents the potential pregnancy-related hormonal and biomechanical changes as well as neuromuscular adaptations that may play a role in the development and intensity of LBPP in pregnant women. Dotted arrows indicate potential modulating factors or hypothetical relationship between two boxes. ${ }^{*}$ The peak of LBPP intensity generally appears between 24 and 36 weeks of pregnancy (4). ${ }^{*}$ Women suffering from LBPP during pregnancy will still experience LBPP beyond 3 months [33\% $(5)]$ and 12 months $[25 \%(3,6)]$ after delivery.

such as changes in pain modulation. Interactions between these various changes and adaptations remain mostly to be investigated. Our research team is currently conducting a prospective laboratory study which aims to characterize the evolution of neuromechanical (lumbar muscle activity and kinematic), physiological (pregnancy-related hormones) and clinical (pain intensity and disability) changes at all pregnancy trimesters. 
The fact that the several physiological changes occurring during pregnancy, including motor behaviors and neuromuscular control, are not systematically associated to LBPP (or the threat of LBPP) suggests that motor adaptations during pregnancy may precede LBPP. However, one must not forget that previous history of LBP is one of the most powerful LBPP predictor. Following delivery, women fully or partially return to pre pregnancy hormonal, biomechanical and neuromuscular state and may or may not experience persistent or recurring LBPP in the post-partum period. Whether or not recovering from pregnancy-related changes and adaptations can explain persisting LBPP remains to be investigated.

\section{CONCLUSION}

In conclusion, we proposed a model for underlying causes of LBPP that should allow clinicians and researchers to consider the multifactorial nature of LBPP and the potentially competing mechanisms (biomechanical, hormonal, and neuromuscular processes) as well as their interactions. It also considers and weighs in current evidence to guide future research. Future research should include observational studies conducted to determine the potential role of individual and combined physical

\section{REFERENCES}

1. Gutke A, Boissonnault J, Brook G, Stuge B. The severity and impact of pelvic girdle pain and low-back pain in pregnancy: a multinational study. J Womens Health. (2018) 27:510 doi: 10.1089/jwh.2017.6342

2. Vleeming A, Albert HB, Ostgaard HC, Sturesson B, Stuge B. European guidelines for the diagnosis and treatment of pelvic girdle pain. Eur Spine J. (2008) 17:794e doi: 10.1007/s00586-008-0602-4

3. Wu WH, Meijer OG, Uegaki K, Mens JM, van Dieen JH, Wuisman PI, et al. Pregnancy-related pelvic girdle pain (PPP), I: terminology, clinical presentation, and prevalence. Eur Spine J. (2004) 13:575e doi: 10.1007/s00586-003-0615-y

4. Kristiansson P, Svardsudd K, von Schoultz B. Reproductive hormones and aminoterminal propeptide of type III procollagen in serum as early markers of pelvic pain during late pregnancy. Am J Obstet Gynecol. (1999) 180:128 doi: 10.1016/S0002-9378(99)70162-6

5. Gutke A, Lundberg M, Ostgaard HC, Oberg B. Impact of postpartum lumbopelvic pain on disability, pain intensity, health-related quality of life, activity level, kinesiophobia, and depressive symptoms. Eur Spine J. (2011) 20:440 doi: 10.1007/s00586-010-1487-6

6. Albert H, Godskesen M, Westergaard J. Prognosis in four syndromes of pregnancy-related pelvic pain. Acta Obstet Gynecol Scand. (2001) 80:505 doi: 10.1080/j.1600-0412.2001.080006505.x

7. Olsson C, Nilsson-Wikmar L. Health-related quality of life and physical ability among pregnant women with and without back pain in late pregnancy. Acta Obstet Gynecol Scand. (2004) 83:351 doi: 10.1080/j.0001-6349.2004.00384.x

8. Mogren I. Perceived health, sick leave, psychosocial situation, and sexual life in women with low-back pain and pelvic pain during pregnancy. Acta Obstet Gynecol Scand. (2006) 85:647 doi: 10.1080/00016340600607297

9. Elden H, Lundgren I, Robertson E. Life's pregnant pause of pain: pregnant women's experiences of pelvic girdle pain related to daily life: a Swedish interview study. Sex Reprod Healthc. (2013) 4:29 doi: 10.1016/j.srhc.2012.11.003

10. Gutke A, Ostgaard HC, Oberg B. Pelvic girdle pain and lumbar pain in pregnancy: a cohort study of the consequences in and physiological adaptations in the development of LBPP in pregnant women.

\section{DATA AVAILABILITY STATEMENT}

The original contributions presented in the study are included in the article/supplementary material, further inquiries can be directed to the corresponding author/s.

\section{AUTHOR CONTRIBUTIONS}

$\mathrm{CD}$, S-MR, and MD contributed to conceptualization. CD, JA, S-MR, and MD designed the model. S-MR and $\mathrm{MD}$ supervised the project. A-AM, S-MR, and $\mathrm{MD}$ revised the manuscript critically. All authors wrote the manuscript and approved the final manuscript.

\section{FUNDING}

Funding for this study was provided by the Chaire de Recherche Internationale en Santé Neuromusculosquelettique and its partner, the Centre Intégré Universitaire de Santé et de Services Sociaux de la Mauricie-et-du-Centre-du-Québec. terms of health and functioning. Spine. (2006) 31:E14931:6 doi: 10.1097/01.brs.0000201259.63363.e1

11. Owe KM, Nystad W, Bo K. Correlates of regular exercise during pregnancy: the Norwegian Mother and Child Cohort Study. Scand J Med Sci Sports. (2009) 19:637 doi: 10.1111/j.1600-0838.2008.00840.x

12. Verstraete EH, Vanderstraeten G, Parewijck W. Pelvic Girdle Pain during or after Pregnancy: a review of recent evidence and a clinical care path proposal. Facts Views Vis ObGyn. (2013) 5:33-43.

13. Bjelland EK, Eberhard-Gran M, Nielsen CS, Eskild A. Age at menarche and pelvic girdle syndrome in pregnancy: a population study of 74973 women. BJOG. (2011) 118:1646-6468 doi: 10.1111/j.1471-0528.2011.03099.x

14. Bjelland EK, Kristiansson P, Nordeng H, Vangen S, Eberhard-Gran M. Hormonal contraception and pelvic girdle pain during pregnancy: a population study of 91,721 pregnancies in the Norwegian Mother and Child Cohort. Hum Reprod. (2013) 28:3134-134:3 doi: 10.1093/humrep/det301

15. Gjestland K, Bo K, Owe KM, Eberhard-Gran M. Do pregnant women follow exercise guidelines? Prevalence data among 3482 women and prediction of low-back pain, pelvic girdle pain and depression. Br J Sports Med. (2013) 47:515 doi: 10.1136/bjsports-2012-091344

16. Reese ME, Casey E. Hormonal influence on the neuromusculoskeletal system in pregnancy. In: Fitzgerald CM, Segal NA, editors. Musculoskeletal Health in Pregnancy and Postpartum: An Evidence-Based Guide for Clinicians. Springer (2015). p. 19-34. doi: 10.1007/978-3-319-14319-4_2

17. Hisaw FL, Zarrow MX. The physiology of relaxin. Vitam Horm. (1950) 8:151 doi: 10.1016/S0083-6729(08)60670-6

18. Dragoo JL, Padrez K, Workman R, Lindsey DP. The effect of relaxin on the female anterior cruciate ligament: analysis of mechanical properties in an animal model. Knee. (2009) 16:69 doi: 10.1016/j.knee.2008.09.005

19. Dehghan F, Haerian BS, Muniandy S, Yusof A, Dragoo JL, Salleh N. The effect of relaxin on the musculoskeletal system. Scand J Med Sci Sports. (2014) 24:e22024:4 doi: 10.1111/sms.12149

20. MacLennan AH, Nicolson R, Green RC, Bath M. Serum relaxin and pelvic pain of pregnancy. Lancet. (1986) 2:243 doi: 10.1016/S0140-6736(86)92069-6

21. Aldabe D, Ribeiro DC, Milosavljevic S, Dawn Bussey M. Pregnancyrelated pelvic girdle pain and its relationship with relaxin levels during 
pregnancy: a systematic review. Eur Spine J. (2012) 21:1769-769:2 doi: 10.1007/s00586-012-2162-x

22. Stables D, Rankin J. Physiology in Childbearing: With Anatomy and Related Biosciences. 3rd ed: Edinburgh; New York, NY: BaillirghBioscien (2010).

23. Yu WD, Panossian V, Hatch JD, Liu SH, Finerman GA. Combined effects of estrogen and progesterone on the anterior cruciate ligament. Clin Orthopaed Relat Res. (2001) 383:268 doi: 10.1097/00003086-200102000-00031

24. Charlton WP, Coslett-Charlton LM, Ciccotti MG. Correlation of estradiol in pregnancy and anterior cruciate ligament laxity. Clin Orthopaed Relat Res. (2001) 165-70. doi: 10.1097/00003086-200106000-00022

25. Blackburn S. Maternal, Fetal, \& Neonatal Physiology - A Clinical Perspective. 3rd ed. Elsevier Health Sciences (2007).

26. Soma-Pillay P, Nelson-Piercy C, Tolppanen H, Mebazaa A. Physiological changes in pregnancy: review articles. Cardiovasc J Afr. (2016) 27:89 doi: 10.5830/CVJA-2016-021

27. Rankin J. Physiology in Childbearing: With Anatomy and Related Biosciences. 4rd ed. Edinburgh; New York, NY: Bailli Yorkoscien (2017).

28. Conder R, Zamani R, Akrami M. The biomechanics of pregnancy: a systematic review. J Funct Morphol Kinesiol. (2019) 4:72. doi: 10.3390/jfmk4040072

29. Whitcome KK, Shapiro LJ, Lieberman DE. Fetal load and the evolution of lumbar lordosis in bipedal hominins. Nature. (2007) 450:1075-0750 doi: $10.1038 /$ nature 06342

30. Betsch M, Wehrle R, Dor L, Rapp W, Jungbluth P, Hakimi M, et al. Spinal posture and pelvic position during pregnancy: a prospective rasterstereographic pilot study. Eur Spine J. (2015) 24:1282-282:5 doi: 10.1007/s00586-014-3521-6

31. Franklin ME, Conner-Kerr T. An analysis of posture and back pain in the first and third trimesters of pregnancy. J Orthop Sports Phys Ther. (1998) 28:133 doi: 10.2519/jospt.1998.28.3.133

32. Glinkowski WM, Tomasik P, Walesiak K, Gluszak M, Krawczak K, Michonski J, et al. Posture and low back pain during pregnancy - 3D study. Ginekol Pol. (2016) 87:575 doi: 10.5603/GP.2016.0047

33. Moore K, Dumas GA, Reid JG. Postural changes associated with pregnancy and their relationship with low-back pain. Clin Biomech. (1990) 5:169 doi: 10.1016/0268-0033(90)90020-7

34. Schroder G, Kundt G, Otte M, Wendig D, Schober HC. Impact of pregnancy on back pain and body posture in women. J Phys Ther Sci. (2016) 28:1199199:6 doi: 10.1589/jpts.28.1199

35. Yoo H, Shin D, Song C. Changes in the spinal curvature, degree of pain, balance ability, and gait ability according to pregnancy period in pregnant and nonpregnant women. J Phys Ther Sci. (2015) 27:279 doi: 10.1589/jpts.27.279

36. Nicholls JA, Grieve DW. Posture, performance and discomfort in pregnancy. Appl Ergon. (1992) 23:128 doi: 10.1016/0003-6870(92)90085-A

37. Okanishi N, Kito N, Akiyama M, Yamamoto M. Spinal curvature and characteristics of postural change in pregnant women. Acta Obstet Gynecol Scand. (2012) 91:856 doi: 10.1111/j.1600-0412.2012.01400.x

38. McCrory JL, Chambers AJ, Daftary A, Redfern MS. The pregnant "waddle": an evaluation of torso kinematics in pregnancy. J Biomech. (2014) 47:2964-964:4 doi: 10.1016/j.jbiomech.2014.07.009

39. Krkeljas Z. Changes in gait and posture as factors of dynamic stability during walking in pregnancy. Hum Mov Sci. (2018) 58:315 doi: 10.1016/j.humov.2017.12.011

40. Bivia-Roig G, Lison JF, Sanchez-Zuriaga D. Changes in trunk posture and muscle responses in standing during pregnancy and postpartum. PLOS ONE. (2018) 13:e0194853. doi: 10.1371/journal.pone.0194853

41. Catena RD, Campbell N, Wolcott WC, Rothwell SA. Anthropometry, standing posture, and body center of mass changes up to 28 weeks postpartum in Caucasians in the United States. Gait Posture. (2019) 70:196 doi: 10.1016/j.gaitpost.2019.03.009

42. Gilleard WL, Crosbie J, Smith R. Static trunk posture in sitting and standing during pregnancy and early postpartum. Arch Phys Med Rehabil. (2002) 83:1739-739:2 doi: 10.1053/apmr.2002.36069

43. Dumas GA, Reid JG, Wolfe LA, Griffin MP, McGrath MJ. Exercise, posture, and back pain during pregnancy. Clin Biomech. (1995) 10:98 doi: 10.1016/0268-0033(95)92046-O

44. Bjorklund K, Nordstrom ML, Bergstrom S. Sonographic assessment of symphyseal joint distention during pregnancy and post partum with special reference to pelvic pain. Acta Obstet Gynecol Scand. (1999) 78:125 doi: 10.1034/j.1600-0412.1999.780210.x

45. Mogren IM, Pohjanen AI. Low back pain and pelvic pain during pregnancy: prevalence and risk factors. Spine. (2005) 30:983 doi: 10.1097/01.brs.0000158957.42198.8e

46. Mogren IM, BMI, pain and hyper-mobility are determinants of long-term outcome for women with low back pain and pelvic pain during pregnancy. Eur Spine J. (2006) 15:1093-093:6 doi: 10.1007/s00586-005-0004-9

47. Berezin D. Pelvic insufficiency during pregnancy and after parturition: a clinical study. Acta Obstet Gynecol Scand. (1954) 33:1 doi: 10.3109/00016345409154960

48. Damen L, Buyruk HM, Guler-Uysal F, Lotgering FK, Snijders CJ, Stam HJ. Pelvic pain during pregnancy is associated with asymmetric laxity of the sacroiliac joints. Acta Obstet Gynecol Scand. (2001) 80:1019-019:1 doi: 10.1034/j.1600-0412.2001.801109.x

49. Albert HB, Godskesen M, Korsholm L, Westergaard JG. Risk factors in developing pregnancy-related pelvic girdle pain. Acta Obstet Gynecol Scand. (2006) 85:539 doi: 10.1080/00016340600578415

50. Pool-Goudzwaard AL, Vleeming A, Stoeckart R, Snijders CJ, Mens JM. Insufficient lumbopelvic stability: a clinical, anatomical and biomechanical approach to 'a-specific' low back pain. Man Ther. (1998) 3:12 doi: 10.1054/math.1998.0311

51. Christensen L, Veierod MB, Vollestad NK, Jakobsen VE, Stuge B, Cabri J, et al. Kinematic and spatiotemporal gait characteristics in pregnant women with pelvic girdle pain, asymptomatic pregnant and non-pregnant women. Clin Biomech. (2019) 68:45 doi: 10.pat1016/j.clinbiomech.2019.05.030

52. Gutke A, Ostgaard HC, Oberg B. Association between muscle function and low back pain in relation to pregnancy. J Rehabil Med. (2008) 40:304 doi: 10.2340/16501977-0170

53. Kerbourc'h F, Bertuit J, Feipel V, Rooze M. Pregnancy and pelvic girdle pain analysis of center of pressure during gait. J Am Podiatr Med Assoc. (2017) 107:299-306. doi: 10.7547/15-087

54. Aldabe D, Milosavljevic S, Bussey MD. Is pregnancy related pelvic girdle pain associated with altered kinematic, kinetic and motor control of the pelvis? A systematic review. Eur Spine J. (2012) 21:1777-777:2 doi: 10.1007/s00586-012-2401-1

55. Ji X, Morino S, Iijima $H$, Ishihara $M$, Kawagoe $M$, Umezaki $F$, et al. The Association of variations in hip and pelvic geometry with pregnancyrelated sacroiliac joint pain based on a longitudinal analysis. Spine. (2019) 44:E67644:9 doi: 10.1097/BRS.0000000000002774

56. To WW, Wong MW. Back pain symptoms and bone mineral density changes in pregnancy as measured by quantitative ultrasound. Gynecol Obstet Invest. (2009) 67:36 doi: 10.1159/000158650

57. Bivia-Roig G, Lison JF, Sanchez-Zuriaga D. Effects of pregnancy on lumbar motion patterns and muscle responses. Spine J. (2019) 19:364 doi: 10.1016/j.spinee.2018.08.009

58. de Groot M, Pool-Goudzwaard AL, Spoor CW, Snijders CJ. The active straight leg raising test (ASLR) in pregnant women: differences in muscle activity and force between patients and healthy subjects. Man Ther. (2008) 13:68 doi: 10.1016/j.math.2006.08.006

59. Pool-Goudzwaard AL, Slieker ten Hove MC, Vierhout ME, Mulder PH, Pool JJ, Snijders CJ, et al. Relations between pregnancy-related low back pain, pelvic floor activity and pelvic floor dysfunction. Int Urogynecol J Pelvic Floor Dysfunct. (2005) 16:468 doi: 10.1007/s00192-005-1292-7

60. Stuge B, Saetre K, Ingeborg Hoff B. The automatic pelvic floor muscle response to the active straight leg raise in cases with pelvic girdle pain and matched controls. Man Ther. (2013) 18:327 doi: 10.1016/j.math.2012.12.004

61. Sihvonen T, Huttunen M, Makkonen M, Airaksinen O. Functional changes in back muscle activity correlate with pain intensity and prediction of low back pain during pregnancy. Arch Phys Med Rehabil. (1998) 79:1210-210:8 doi: 10.1016/S0003-9993(98)90264-7

62. Stuge B, Saetre K, Braekken IH. The association between pelvic floor muscle function and pelvic girdle pain-a matched case control 3D ultrasound study. Man Ther. (2012) 17:150 10.1016/j.math.2011.12.004

63. Gold M, Gebhart G. Peripheral Pain Mechanisms and Nociceptor Sensitization. Bonica's Pain Management. Lippincott Williams \& Wilkins (LWW) (2010). p. $25-34$. 
64. Graven-Nielsen T, Arendt-Nielsen L. Assessment of mechanisms in localized and widespread musculoskeletal pain. Nat Rev Rheumatol. (2010) 6:599 10.1038/nrrheum.2010.107

65. Jankowski MP, Koerber HR. Neurotrophic factors and nociceptor sensitization. Transl Pain Res Mouse Man. (2010) 31:50. doi: 10.1201/9781439812105-c2

66. Melchior M, Poisbeau P, Gaumond I, Marchand S. Insights into the mechanisms and the emergence of sex-differences in pain. Neuroscience. (2016) 338:63 10.1016/j.neuroscience.2016.05.007

67. Popescu A, LeResche L, Truelove EL, Drangsholt MT. Gender differences in pain modulation by diffuse noxious inhibitory controls: a systematic review. Pain. (2010) 150:309, 10.1016/j.pain.2010.05.013

68. Traub RJ Ji Y. Sex differences and hormonal modulation of deep tissue pain. Front Neuroendocrinol. (2013) 34:350 10.1016/j.yfrne.2013.07.002

69. Saito S, Nakashima A, Shima T, Ito M. Th1/Th2/Th17 and regulatory T-cell paradigm in pregnancy. Am J Reprod Immunol. (2010) 63:601 rod. doi: 10.1111/j.1600-0897.2010.00852.x

70. Amandusson. paradigm in pregnancy ulation of deep tissueFront Neuroendocrinol. (2013) 34:329 doi: 10.1016/j.yfrne.2013.06.001

71. Gintzler AR. Endorphin-mediated increases in pain threshold during pregnancy. Science. (1980) 210:193 doi: 10.1126/science.7414330

72. Jarvis S, McLean KA, Chirnside J, Deans LA, Calvert SK, Molony $\mathrm{V}$, et al. Opioid-mediated changes in nociceptive threshold during pregnancy and parturition in the sow. Pain. (1997) 72:153 doi: 10.1016/S0304-3959(97)00027-4

73. Ohel I, Walfisch A, Shitenberg D, Sheiner E, Hallak M, A. rise in pain threshold during labor: a prospective clinical trial. Pain. (2007) 132(Suppl. 1):S104ppl7, doi: 10.1016/j.pain.2007.05.007

74. Dunbar AH, Price DD, Newton RA. An assessment of pain responses to thermal stimuli during stages of pregnancy. Pain. (1988) $35: 265 \mathrm{H}$, doi: 10.1016/0304-3959(88)90136-4

75. Carvalho B, Granot M, Sultan P, Wilson H, Landau R. A Longitudinal study to evaluate pregnancy-induced endogenous analgesia and pain modulation. Reg Anesth Pain Med. (2016) 41:175th doi: 10.1097/AAP.0000000000000359

76. Goolkasian P, Rimer BA. Pain reactions in pregnant women. Pain. (1984) 20:87 doi: 10.1016/0304-3959(84)90814-5

77. Rejano-Campo M, Desvergee A, Pizzoferrato AC. Relationship between perineal characteristics and symptoms and pelvic girdle pain: a literature review. Prog Urol. (2018) 28:193 doi: 10.1016/j.purol.2017.12.007

78. Staikou C, Siafaka I, Petropoulos G, Katafigioti A, Fassoulaki A. Responses to mechanical and electrical stimuli are not attenuated by late pregnancy. Acta Anaesthesiol Belg. (2006) 57:277.

79. Palsson TS, Beales D, Slater H, O’Sullivan P, Graven-Nielsen T. Pregnancy is characterized by widespread deep-tissue hypersensitivity independent of lumbopelvic pain intensity, a facilitated response to manual orthopedic tests, and poorer self-reported health. J Pain. (2015) 16:270TS, doi: 10.1016/j.jpain.2014.12.002

80. Olsson C, Buer N, Holm K, Nilsson-Wikmar L. Lumbopelvic pain associated with catastrophizing and fear-avoidance beliefs in early pregnancy. Acta Obstet Gynecol Scand. (2009) 88:378 doi: 10.1080/00016340902763210
81. Fakari FR, Simbar M, Naz MSG. The relationship between fear-avoidance beliefs and pain in pregnant women with pelvic girdle pain: a cross-sectional study. Int J Commun Based Nurs Midwifery. (2018) 6:305-13.

82. Greenspan JD, Craft RM, LeResche L, Arendt-Nielsen L, Berkley KJ, Fillingim RB, et al. Studying sex and gender differences in pain and analgesia: a consensus report. Pain. (2007) 132(Suppl. 1):S262ppl7 doi: 10.1016/j.pain.2007.10.014

83. Frolich MA, Banks C, Warren W, Robbins M, Ness T. The association between progesterone, estradiol, and oxytocin and heat pain measures in pregnancy: an observational cohort study. Anesth Analg. (2016) 123:396 doi: 10.1213/ANE.0000000000001259

84. Street LM, Harris L, Curry RS, Eisenach JC. Capsaicin-induced pain and sensitisation in the postpartum period. Br J Anaesth. (2019) 122:103 doi: 10.1016/j.bja.2018.09.026

85. Deering R, Pashibin T, Cruz M, Hunter SK, Hoeger Bement M. Fatiguing trunk flexor exercise decreases pain sensitivity in postpartum women. Front Physiol. (2019) 10:315. doi: 10.3389/fphys.2019.00315

86. Gutierrez S, Liu B, Hayashida K-i, Houle TT, Eisenach JC. Reversal of peripheral nerve injury-induced hypersensitivity in the postpartum period: role of spinal oxytocin. J Am Soc Anesthesiol. (2013) 118:152 doi: 10.1097/ALN.0b013e318278cd21

87. Hodges PW, Tucker K. Moving differently in pain: a new theory to explain the adaptation to pain. Pain. (2011) 152(Suppl. 3):S908. doi: $10.1016 /$ j.pain.2010.10.020

88. Hodges PW. Pain and motor control: from the laboratory to rehabilitation. $J$ Electromyogr Kinesiol. (2011) 21:220 doi: 10.1016/j.jelekin.2011.01.002

89. Hodges PW, Coppieters MW, MacDonald D, Cholewicki J. New insight into motor adaptation to pain revealed by a combination of modelling and empirical approaches. Eur J Pain. (2013) 17:1138-138:3 doi: 10.1002/j.1532-2149.2013.00286.x

Conflict of Interest: The authors declare that the research was conducted in the absence of any commercial or financial relationships that could be construed as a potential conflict of interest.

Publisher's Note: All claims expressed in this article are solely those of the authors and do not necessarily represent those of their affiliated organizations, or those of the publisher, the editors and the reviewers. Any product that may be evaluated in this article, or claim that may be made by its manufacturer, is not guaranteed or endorsed by the publisher.

Copyright (c) 2021 Daneau, Abboud, Marchand, Houle, Pasquier, Ruchat and Descarreaux. This is an open-access article distributed under the terms of the Creative Commons Attribution License (CC BY). The use, distribution or reproduction in other forums is permitted, provided the original author(s) and the copyright owner(s) are credited and that the original publication in this journal is cited, in accordance with accepted academic practice. No use, distribution or reproduction is permitted which does not comply with these terms. 